\title{
Efectos de una intervención quirúrgica menor sobre la calidad de vida en niños desde diferentes perspectivas
}

Javier Brazo-Sayavera ${ }^{1,2}$, Inés M. Parejo-Cabezas ${ }^{3}$, Narcís Gusi' y Pedro R. Olivares ${ }^{2,4}$

${ }^{1}$ Facultad de Ciencias del Deporte, Universidad de Extremadura, Caceres, España; ${ }^{2}$ Instituto Superior de Educación Física, Universidad de la República, Rivera, Uruguay; ${ }^{3}$ Complejo Hospitalario San Pedro de Alcántara, Cáceres, España; ${ }^{4}$ Instituto de Actividad Física y Salud, Universidad Autónoma de Chile, Talca, Chile

\section{Resumen}

Objetivo: Comparar la percepción de la calidad de vida relacionada con la salud (CVRS) de padres y médico con respecto al paciente en población infantil sometida a una intervención quirúrgica menor, y analizar la progresión de la CVRS tras un mes de someterse a la intervención. Método: Participaron 36 pacientes sometidos a intervenciones quirúrgicas menores. Se les administró el cuestionario EQ-5D-Y, y su versión proxy correspondiente a los padres y al médico. Se utilizó la variable TTO. Resultados: Los pacientes refirieron un peor estado de salud, sobre todo en la dimensión de preocupación/tristeza, que los médicos en el preoperatorio $(p<0.05)$, pero la percepción postoperatoria fue similar en pacientes y adultos, atribuible al efecto techo por buena salud postoperatoria. Conclusiones: Los pacientes pediátricos sometidos a cirugía menor mejoran su CVRS tras la intervención, y esta mejora se mantiene tras un mes de la cirugía.

PALABRAS CLAVE: EQ-5D-Y. Pediatría. Cirugía menor.

\begin{abstract}
Objective: To compare the health related quality of life (HRQoL) perceptions in parents and physician regarding the patient undergoing ambulatory surgery, and to analyse the evolution of HRQoL before and after the ambulatory surgery. Method: 36 patients underwent ambulatory surgical interventions taken part in this study. The questionnaires EQ-5D-Y were administered to the patients and questionnaires EQ-5D-Y proxy to parents and physicians. Variable TTO was used. Results: Statistical differences were found at baseline between answers of patients and physicians $(p<0.05)$. However, after surgery, answers of patients and proxies were similar. Conclusions: Paediatric patients undergone to a minor surgery improve HRQoL after the operation and this improvement goes on a month after the surgery.
\end{abstract}

KEY WORDS: EQ-5D-Y. Paediatrics. Minor surgery. 


\section{Introducción}

Las intervenciones quirúrgicas en niños menores de 14 años tienen una incidencia aproximada del $2.33 \%$ de la población pediátrica del área de salud de Cáceres, que da cobertura a 27,000 usuarios en ese intervalo de edad, siendo el Complejo Hospitalario de Cáceres el centro de referencia de cirugía pediátrica para esta y de cirugía plástica infantil para toda la Comunidad Autónoma de Extremadura. El número de intervenciones quirúrgicas programadas que se realizaron en el año 2013 en niños menores de 14 años fue de 631 entre todas las especialidades, de las cuales el $99 \%$ fueron intervenciones quirúrgicas menores (Fuente: Unidad de Codificación del Complejo Hospitalario de Cáceres). Se entiende por intervenciones quirúrgicas menores los procedimientos quirúrgicos sencillos, de corta duración, que requieren como mucho un día de ingreso hospitalario y con una incidencia de complicaciones postoperatorias muy baja'1. Las patologías más frecuentes que se intervienen en este rango de edad suelen ser fimosis, criptorquidia, hidrocele, hernia inguinal, frenillo lingual, orejas aladas, adenoiditis, amigdalitis y lesiones de la piel y del tejido subcutáneo.

La calidad de vida relacionada con la salud (CVRS) ha sido empleada ampliamente como medida de efectividad de procedimientos quirúrgicos ${ }^{2-4}$, y en un paciente representa el impacto que una enfermedad y la aplicación de su tratamiento tienen sobre la percepción del paciente en su satisfacción y bienestar físico, psíquico, social y espiritual. La CVRS se considera cada vez más un área importante de la investigación en niños y adolescentes, lo que justifica la cantidad de estudios que están apareciendo sobre el tema en la actualidad con el fin de conocer los efectos que las enfermedades tienen en la vida cotidiana de los pacientes que sufren enfermedades crónicas $^{5-8}$.

Algunos estudios han analizado la CVRS en niños tras someterse a intervenciones quirúrgicas. Recientemente, han sido varios autores los que han estudiado la CVRS en niños sometidos a otoplastias, demostrando que tras la intervención presentaban una mejora en la CVRS $^{9-11}$. En relación con otras enfermedades, se ha analizado la CVRS en niños con síndrome de apnea-hipopnea del sueño a los que se les intervino de adenoamigdalectomía, y se concluyó que los niños mejoran la CVRS tras la intervención, sobre todos aquellos cuya enfermedad es más grave previamente ${ }^{4}$. Otra patología de la infancia en la que también ha sido estudiada la CVRS ha sido la cirugía del estrabismo. La CVRS se ha empleado para determinar la viabilidad de la intervención quirúrgica ${ }^{12}$ o bien para analizar a qué se debía la pérdida de CVRS en niños con estrabismo ${ }^{13}$.

El EQ-5D es uno de los instrumentos de evaluación de la CVRS más utilizados en el mundo, ha demostrado tener una buena fiabilidad en el análisis de la CVRS de pacientes sometidos a cirugía ${ }^{14,15}$ y además posibilita la realización de análisis de coste-utilidad. EI EQ-5D cuenta con una versión específica para su uso en población infantil y adolescente, denominada $E Q-5 D-Y^{16}$, que está validada también en español ${ }^{17}$. Además, este instrumento cuenta con una versión proxy que permite evaluar la CVRS de la población infantil y adolescente con dificultades físicas o psíquicas, o que aún no sabe leer, a través de sus padres, tutores, médicos, cuidadores, etc. ${ }^{18}$.

La información sobre la CVRS de los niños desde el punto de vista de la familia es determinante al tomar decisiones médicas, ya que los padres tienen un papel importante en la definición de lo que ellos creen que es mejor para sus hijos y en la evaluación del éxito del tratamiento. Cada perspectiva proxy tiene el potencial de proporcionar una información valiosa que puede converger o divergir en mayor o menor medida de la autoevaluación de la CVRS por el paciente ${ }^{19}$. Sin embargo, principalmente en el caso de los niños, la información proporcionada puede ser menos consistente que la ofrecida por sus padres, en particular en los estudios longitudinales, debido a los cambios en las actitudes, habilidades y prioridades, propios del proceso de desarrollo normal de los niños ${ }^{20}$.

Dada la importancia de la CVRS y la escasez de recursos disponibles para una demanda creciente de servicios sanitarios ${ }^{21}$, surge la necesidad de investigar el efecto de las intervenciones quirúrgicas menores sobre la CVRS en niños con instrumentos genéricos como el EQ-5D-Y, cuya validez y fiabilidad han sido demostradas ${ }^{22}$. Del mismo modo, es necesario contar con la opinión de los padres y del personal médico en este tipo de evaluaciones. Además, la versión proxy del EQ-5D-Y aún no ha sido utilizada en estudios Iongitudinales, por lo que se necesitan estudios de este tipo para comprobar su sensibilidad.

El objetivo del presente estudio es analizar los efectos sobre la CVRS en pacientes pediátricos sometidos a intervenciones quirúrgicas menores, comparando la percepción del paciente con la de los proxys más comunes: padres, madres y médicos. 


\section{Método}

Los participantes de este estudio fueron pacientes pediátricos que se sometieron a una intervención quirúrgica menor en el Hospital de Cáceres durante el año 2014. Finalmente, 37 pacientes (25 niños y 11 niñas) con edades comprendidas entre los 8 y los 12 años (10.31 \pm 1.35 años), intervenidos de cirugía menor, fueron incluidos en el presente estudio. Un caso fue excluido por no poder aportar el cuestionario del padre.

El estudio fue aprobado por el Comité de Bioética y Bioseguridad de la Universidad de Extremadura. Todos los participantes firmaron un consentimiento informado, que cumplía con los principios de la declaración de Helsinki.

Se utilizaron los siguientes instrumentos:

- Variables sociodemográficas: se recogieron datos de edad, sexo, nivel educativo y situación migratoria en una hoja de registro.

- EQ-5D-Y: adaptado del EQ-5D para adultos, validado por Wille, et al. ${ }^{16} \mathrm{y}$ adaptado al español por Gusi, et al. ${ }^{17}$, se trata de un instrumento genérico para medir la CVRS en niños y adolescentes de una manera apropiada para su edad. Es de fácil comprensión, muy corto, con bajo número de respuestas perdidas o equivocadas, y se cumplimenta en un tiempo aproximado de 3 minutos por persona ${ }^{23}$. Consta de una escala analógica visual que abarca una puntuación de 0 a 100, siendo 0 el peor estado de salud y 100 el mejor estado, e incluye cinco dimensiones, que son movilidad, autocuidado, actividades de la vida diaria, dolor/malestar y ansiedad/depresión. Cada dimensión tiene tres posibles respuestas o niveles de problema que presentan en esa dimensión (ausencia que se codifica con 1, moderado que se codifica con 2 o grave que se codifica con 3$)^{24}$. El niño debe marcar el nivel de problemas, dentro de cada dimensión, que mejor describa su estado de salud en ese día. Así, se definen 243 «estados de salud» diferentes, siendo el 11111 el mejor estado de salud posible. Los estados de salud EQ-5D pueden convertirse en un índice-resumen, tarifa o utilidad aplicando una fórmula de regresión basada en el valor comparativo ofrecido por la población general a cada estado de salud. En este estudio se ha utilizado la tarifa temporal denominada TTO (time-trade-off) ${ }^{25}$, que abarca valores de -0.59 a 1 , siendo el valor 1 el estado de salud plena y 0 la muerte.

- EQ-5D-Y proxy: adaptado al español por Olivares, et al. ${ }^{18}$, este cuestionario ha demostrado ser claro y comprensible por parte de los padres y madres de niños y adolescentes de la población general. Es un instrumento similar al EQ-5D-Y, pero adaptado y validado para su uso en proxy ${ }^{26}$. El cuestionario se cumplimentó a partir de la percepción que los padres y el médico tuvieron sobre la calidad de vida del paciente.

El cuestionario EQ-5D-Y fue administrado a los pacientes, y el EQ-5D-Y proxy fue administrado al padre, a la madre y al médico responsable de la intervención.

Un miembro del equipo investigador se puso en contacto con el representante legal del paciente previamente a la intervención quirúrgica. Le informó de manera conveniente de los objetivos y del protocolo del estudio, y obtuvo la firma del consentimiento informado.

Se suministraron los cuestionarios, que cumplimentaron en presencia de un miembro del equipo investigador antes de la intervención quirúrgica. Del mismo modo, se facilitó el mismo cuestionario en las 24 horas posteriores a la intervención, una vez que el paciente se encontraba ya en la planta de hospitalización. Se contactó telefónicamente con los participantes un mes después de la intervención con el objetivo de que volviesen a contestar al mismo cuestionario. La tasa de respuesta fue del $100 \%$.

Para el tratamiento y el almacenamiento de los datos se utilizó el paquete estadístico SPSS versión 20.0 (IBM Corporation, Armonk: NY, EE.UU). Se realizaron la prueba Shapiro-Wilks para comprobar la normalidad de las variables, la prueba de homocedasticidad de Levene y la prueba de aleatoriedad de Rachas, además de calcular los estadísticos descriptivos básicos, media y desviación típica.

Para comprobar el grado de coincidencia de las respuestas de niños, padres y médicos, se llevó a cabo la prueba de concordancia de Kendall. Esta prueba ofrece valores de 0 a 1 , siendo 1 un acuerdo total y 0 un desacuerdo total.

Para evaluar la evolución del estado de salud percibido por los pacientes, y debido a las características de los datos, que adolecían de homocedasticidad, se llevó a cabo la prueba no paramétrica de Friedman. Esta prueba puede considerarse como la alternativa no paramétrica al ANOVA de medidas repetidas. El nivel de significación fue $p \leq 0.05$.

Adicionalmente, se calculó el tamaño del efecto mediante la d de Cohen, ${ }^{27}$ utilizando la siguiente 
ecuación matemática para cada variable entre antes y después de la evaluación:

$$
\frac{\left(\text { Media }_{1}-\text { Media }_{2}\right)}{\left\{\left(D T_{1}-D T_{2}\right)\right\}^{2}}
$$

donde media ${ }_{1}$ y desviación típica ${ }_{1}$ representan valores de la evaluación inicial, y media ${ }_{2}$ y desviación típi$\mathrm{ca}_{2}$ valores de la evaluación final. Los valores que adquiría este parámetro fueron clasificados como ligero, moderado y gran efecto para $0.20,0.50$ y 0.80 , respectivamente. El cálculo del tamaño del efecto permite observar cuán grande ha sido la variación obtenida en los resultados, independientemente de su significación estadística.

\section{Resultados}

De los 36 participantes incluidos en el análisis, 11 eran niñas y 25 eran niños, con un rango de edad de 8 a 12 años.

En relación con los problemas indicados en el EQ5D-Y (Tabla 1), se observa que tan solo se han indicado problemas en las dimensiones de dolor/malestar y sentirse preocupado, triste 0 infeliz, obteniendo un efecto techo del 63.9 , el 77.8 , el 77.8 y el $86.1 \%$ en las respuestas de los niños, las madres, los padres y los médicos, respectivamente.

Se observa que no existe concordancia ( $p<0.001$ ) sobre el estado de salud del paciente entre las respuestas obtenidas por el propio paciente y los proxy de los adultos (padre, madre y médico) en el preoperatorio (Tabla 2). El análisis de comparación por pares indica que esto se debe a las diferencias en los resultados de los niños y los médicos $(p=0.045)$, existiendo concordancia en las comparaciones entre los niños y las madres y los padres ( $p=0.144$ en ambos). Tanto en el postoperatorio como en la evaluación tras un mes de la intervención, se encontró concordancia en los resultados de los niños y todos los proxy ( $p=0.392$ en ambas mediciones).

En el análisis sobre los efectos de la intervención (Tabla 3) se observan diferencias estadísticamente significativas entre las diferentes evaluaciones realizadas en el grupo de pacientes (a las 24 horas y al mes de la intervención quirúrgica). En cuanto a los proxy (padres, madres y médico), no presentan diferencias estadísticamente significativas en su percepción de la CVRS del paciente entre la línea base y el resto de las evaluaciones. Sin embargo, el tamaño del efecto es moderado en los grupos de padres y madres en las dos evaluaciones postoperatorias con respecto a la
Tabla 1. Porcentaje de problemas indicados en el EQ-5D-Y

\begin{tabular}{|c|c|c|c|c|c|c|c|c|}
\hline & \multicolumn{2}{|c|}{$\begin{array}{c}\text { Niños } \\
(n=36)\end{array}$} & \multicolumn{2}{|c|}{$\begin{array}{l}\text { Madres } \\
(n=36)\end{array}$} & \multicolumn{2}{|c|}{$\begin{array}{l}\text { Padres } \\
(n=36)\end{array}$} & \multicolumn{2}{|c|}{$\begin{array}{c}\text { Médicos } \\
(n=36)\end{array}$} \\
\hline & $\%$ & $\mathrm{n}$ & $\%$ & $\mathbf{n}$ & $\%$ & $\mathbf{n}$ & $\%$ & $\mathrm{n}$ \\
\hline \multicolumn{9}{|l|}{ Movilidad } \\
\hline Ningún problema & 100 & 36 & 100 & 36 & 100 & 36 & 100 & 36 \\
\hline $\begin{array}{l}\text { Algunos/muchos } \\
\text { problemas }\end{array}$ & & & & & & & & \\
\hline $\begin{array}{l}\text { problemas } \\
\text { Valores perdidos }\end{array}$ & 0 & $\begin{array}{l}0 \\
0\end{array}$ & 0 & $\begin{array}{l}0 \\
0\end{array}$ & $\begin{array}{l}0 \\
0\end{array}$ & $\begin{array}{l}0 \\
0\end{array}$ & $\begin{array}{l}0 \\
0\end{array}$ & $\begin{array}{l}0 \\
0\end{array}$ \\
\hline \multicolumn{9}{|l|}{ Autocuidado } \\
\hline Ningún problema & 100 & 36 & 100 & 36 & 100 & 36 & 100 & 36 \\
\hline Algunos/muchos & & & & & & & & \\
\hline problemas & 0 & 0 & 0 & 0 & 0 & 0 & 0 & 0 \\
\hline Valores perdidos & 0 & 0 & 0 & 0 & 0 & 0 & 0 & 0 \\
\hline \multicolumn{9}{|c|}{ Realizar actividades habituales } \\
\hline Ningún problema & 97.2 & 35 & 100 & 36 & 100 & 36 & 100 & 36 \\
\hline Algunos problemas & 2.8 & 1 & 0 & 0 & 0 & 0 & 0 & 0 \\
\hline Muchos problemas & 0 & 0 & 0 & 0 & 0 & 0 & 0 & 0 \\
\hline Valores perdidos & 0 & 0 & 0 & 0 & 0 & 0 & 0 & 0 \\
\hline \multicolumn{9}{|l|}{ Tener dolor o malestar } \\
\hline Ningún problema & 75 & 27 & 88.9 & 32 & 88.9 & 32 & 97.2 & 35 \\
\hline Algunos problemas & 25 & 9 & 11.1 & 4 & 11.1 & 4 & 2.8 & 1 \\
\hline Muchos problemas & 0 & 0 & 0 & 0 & 0 & 0 & 0 & 0 \\
\hline Valores perdidos & 0 & 0 & 0 & 0 & 0 & 0 & 0 & 0 \\
\hline \multicolumn{9}{|c|}{ Sentirse preocupado, triste o infeliz } \\
\hline Ningún problema & 83.3 & 30 & 86.1 & 31 & 86.1 & 31 & 86.1 & 31 \\
\hline Un poco & 11.1 & 4 & 13.9 & 5 & 13.9 & 5 & 13.9 & 5 \\
\hline Mucho & 5.6 & 2 & 0 & 0 & 0 & 0 & 0 & 0 \\
\hline Valores perdidos & 0 & 0 & 0 & 0 & 0 & 0 & 0 & 0 \\
\hline
\end{tabular}

Tabla 2. Concordancia en la variable TTO desde los puntos de vista del paciente y distintos proxy antes y después de una intervención quirúrgica menor

\begin{tabular}{lccc}
\hline & Línea base & Antes & Seguimiento tras 1 mes \\
\hline $\mathrm{N}$ & 36 & 36 & 36 \\
$\mathrm{~W}$ de Kendall & 0.225 & 0.028 & 0.028 \\
Sig. asintótica & $<0.001$ & 0.392 & 0.392 \\
\hline
\end{tabular}

línea base, por lo que es posible que con un número mayor de participantes estas diferencias llegasen a ser estadísticamente significativas. Y por último, en los médicos el tamaño del efecto es bajo entre la línea base y los dos momentos del postoperatorio, lo que indica una menor sensibilidad del instrumento utilizado para evaluar los cambios en la CVRS del paciente a través del médico.

\section{Discusión}

El presente estudio muestra que las intervenciones quirúrgicas menores en pacientes pediátricos provocan 
Tabla 3. Efectos de una intervención quirúrgica menor sobre la calidad de vida relacionada con la salud percibida por el paciente y por distintos proxy

\begin{tabular}{lccccc}
\hline & $\begin{array}{c}\text { Línea base } \\
(\mathbf{A})\end{array}$ & $\begin{array}{c}\text { Antes } \\
\mathbf{( B )}\end{array}$ & $\begin{array}{c}\text { Al mes } \\
(\mathbf{C})\end{array}$ & $\begin{array}{c}\text { TE } \boldsymbol{d} \\
(\mathbf{A}-\mathbf{B})\end{array}$ & $\begin{array}{c}\text { TE } \boldsymbol{d} \\
(\mathbf{A}-\mathbf{C})\end{array}$ \\
\hline Pacientes $(\mathrm{n}=36)$ & $0.9337 \pm 0.1232$ & $0.9918 \pm 0.0350^{*}$ & $0.9918 \pm 0.0350^{*}$ & 0.73 & 0.73 \\
Madres $(\mathrm{n}=36)$ & $0.9749 \pm 0.0494$ & $0.9918 \pm 0.0350$ & $0.9918 \pm 0.0350$ & 0.40 & 0.40 \\
Padres $(\mathrm{n}=36)$ & $0.9749 \pm 0.0494$ & $0.9918 \pm 0.0350$ & $0.9918 \pm 0.0350$ & 0.40 & 0.40 \\
Médicos $(\mathrm{n}=36)$ & $0.9851 \pm 0.0396$ & $0.9952 \pm 0.0289$ & $0.9952 \pm 0.0289$ & 0.29 & 0.29 \\
\hline
\end{tabular}

*Diferencia estadísticamente significativa con respecto a la línea base.

TE d: tamaño del efecto.

una mejora en la percepción de su CVRS, evaluada con el cuestionario EQ-5D-Y.

Cuando se compara la percepción de la CVRS del paciente evaluada a través de sus proxy con respecto a la ofrecida por el propio paciente se encuentran diferencias en la fase preoperatoria, mientras que no ocurre lo mismo tras la operación. Estas diferencias fueron mayores en la dimensión sentirse preocupado o triste. Es decir, cuando existe más afectación del estado de salud del paciente, menor concordancia hay entre la CVRS autoevaluada y la CVRS evaluada a través de proxy, coincidiendo con estudios previos en los que se ha observado que la concordancia entre paciente y proxy era menor cuanto mayor era la gravedad de la enfermedad estudiada ${ }^{19}$. Esta menor variabilidad en los estados de salud con menor afectación puede atribuirse parcialmente a un mayor efecto techo, es decir, cuando no hay problemas por el tipo de problema de salud las discrepancias son menores que cuando hay que graduar el nivel o la intensidad del problema. Por otro lado, se ha analizado si el factor depresión contribuye a un menor acuerdo entre paciente y proxy, identificando que este factor producía un menor acuerdo entre estos en población de edad avanzada ${ }^{28}$, por lo que no es adecuado compararlo con lo encontrado en el presente estudio, en el que la quinta dimensión se corresponde con estar preocupado o triste. Sin embargo, los resultados obtenidos concuerdan, ya que las dimensiones en las que se han obtenido diferentes respuestas entre pacientes y proxy han sido las de dolor/malestar y sentirse preocupado, triste o infeliz. En la misma línea que lo encontrado en el presente trabajo, existen otros estudios que han utilizado los cuestionarios proxy $^{29,30}$ y señalan que la mayoría de los pacientes suele tener puntuaciones más bajas que los proxy, es decir, que los pacientes suelen ser más pesimistas en sus respuestas con respecto a su estado de salud que los proxy. Además, sentirse preocupado puede deberse por completo o parcialmente a la causa de la intervención quirúrgica o al afrontamiento de la operación en sí misma. En todo caso, hay que tener en cuenta que la variación de los resultados puede deberse al tamaño de la muestra, la edad de los pacientes, diferencias en la salud previa, etc. ${ }^{31}$. Por ello, el cuestionario proxy puede sustituir la evaluación del paciente, reforzar la información proporcionada por el mismo o bien complementarla. De esta manera, la evaluación proxy de la CVRS puede ayudar a resolver un conflicto en la toma de decisiones médicas, pero es importante señalar que los evaluadores proxy también pueden contribuir a generar conflictos ${ }^{19}$.

En cuanto a la evolución que experimenta la CVRS a lo largo del tiempo analizado, se ha observado que los pacientes intervenidos de cirugías menores experimentan una mejora de la CVRS en las primeras 24 horas de postoperatorio, y el nuevo estado de salud se mantiene a lo largo del primer mes de postoperatorio. Sin embargo, en la CVRS evaluada a través de los proxy no se han encontrado diferencias estadísticamente significativas tras la operación, lo que indica que la sensibilidad al cambio del instrumento que evalúa directamente al paciente es mayor que la de su versión proxy. Se necesitan futuros estudios que analicen la sensibilidad al cambio del EQ-5D-Y proxy con pacientes que se sometan a intervenciones mayores que las analizadas en este estudio. Con esto, además, se solventaría una de las principales limitaciones del presente estudio, que es el efecto techo encontrado en las respuestas del cuestionario. Esto indica que las patologías analizadas apenas afectan a la CVRS en la fase preoperatoria de los pacientes, lo cual limita también la sensibilidad del instrumento en esta población. Este mismo problema se encuentra con este instrumento en población general, en la que se recomienda que se complemente con otros instrumentos más sensibles ${ }^{26}$. Adicionalmente, la generalización de resultados a otro tipo de cirugías mayores debe ser limitada porque 
puede haber variaciones con respecto a las analizadas en el presente estudio. El tamaño muestral puede haber limitado la obtención de significación estadística en los resultados hallados sobre los efectos de la cirugía sobre la CVRS evaluada mediante proxy. Esto se debe a que, aunque las diferencias no eran significativas, el tamaño del efecto fue moderado en los resultados tanto de madres como de padres, lo que indica que con una muestra mayor quizás podrían obtenerse diferencias estadísticamente significativas.

En conclusión, el presente estudio sugiere que la CVRS de pacientes pediátricos sometidos a cirugía menor mejora tras la intervención, y que esta mejora se mantiene un mes después de la cirugía. Adicionalmente se ha encontrado que existe asociación entre las respuestas del paciente y los padres, las madres y los médicos utilizando el EQ-5D-Y y su versión proxy, aunque la sensibilidad al cambio es mayor en la versión autoadministrada.

\section{Conflictos de intereses}

Los autores declaran no tener ningún conflicto de intereses.

\section{Bibliografía}

1. Massa H, Hubert S, Carles M, et al. Anesthésie du patient ambulatoire. Paris: Elsevier Masson; 2010.

2. Bermueller C, Kirsche H, Sebert A, et al. Quality of life and patients' satisfaction after otoplasty. Eur Arch Otorhinolaryngol. 2012;269:2423-31.

3. Kanmaz A, Muderris T, Bercin S, et al. Children's quality of life after adenotonsillectomy. B-Ent. 2013;9:293-8.

4. Lee CH, Kang KT, Weng WC, et al. Quality of life after adenotonsillectomy for children with sleep-disordered breathing: a linear mixed model analysis. Int J Pediatr Otorhinolaryngol. 2014;78:1374-80.

5. Jansson KA, Granath F. Health-related quality of life (EQ-5D) before and after orthopedic surgery. Acta Orthop. 2011;82:82-9.

6. Jansson KA, Nemeth G, Granath F, et al. Health-related quality of life (EQ-5D) before and one year after surgery for lumbar spinal stenosis. $J$ Bone Joint Surg Br. 2009;91:210-6.

7. Werner $H$, Latal $B$, Valsangiacomo Buechel $E$, et al. Health-related quality of life after open-heart surgery. J Pediatr. 2014;164:254-8 e1.

8. Becerra Fontal JA, Bago Granell J, Garre Olmo J, et al. Evaluation of health-related quality of life in patients candidate for spine and other musculoskeletal surgery. Eur Spine J. 2013;22:1002-9.
9. Braun $T$, Hainzinger $T$, Stelter $K$, et al. Health-related quality of life, patient benefit, and clinical outcome after otoplasty using suture techniques in 62 children and adults. Plast Reconstr Surg. 2010;126:2115-24.

10. Hao W, Chorney JM, Bezuhly M, et al. Analysis of health-related quality-of-life outcomes and their predictive factors in pediatric patients who undergo otoplasty. Plast Reconstr Surg. 2013;132:811e-7e.

11. Songu $M$, Kutlu A. Health-related quality of life outcome of children with prominent ears after otoplasty. Eur Arch Otorhinolaryngol. 2014;271:1829-32

12. Yamada T, Hatt SR, Leske DA, et al. Health-related quality of life in parents of children with intermittent exotropia. J AAPOS. 2011;15:135-9.

13. Yamada T, Hatt SR, Leske DA, et al. Specific health-related quality of life concerns in children with intermittent exotropia. Strabismus. 2012;20:145-51.

14. Klassen A, Fitzpatrick R, Jenkinson $C$, et al. Contrasting evidence of the effectiveness of cosmetic surgery from two health related quality of life measures. J Epidemiol Community Health. 1999;53:440-1.

15. Solberg TK, Olsen JA, Ingebrigtsen T, et al. Health-related quality of life assessment by the EuroQol-5D can provide cost-utility data in the field of low-back surgery. Eur Spine J. 2005;14:1000-7.

16. Wille N, Badia X, Bonsel G, et al. Development of the EQ-5D-Y: a child-friendly version of the EQ-5D. Qual Life Res. 2010;19:875-86.

17. Gusi N, Badia X, Herdman M, et al. Traducción y adaptación cultural de la versión española del cuestionario EQ-5D-Y en niños y adolescentes. Aten Primaria. 2009;41:19-23.

18. Olivares PR, Pérez-Sousa MA, Gozalo-Delgado M, et al. Traducción y adaptación cultural al español de la versión Proxy del cuestionario EQ5D-Y. An Pediatr (Barc). 2013;79:157-61.

19. Pickard AS, Knight SJ. Proxy evaluation of health-related quality of life: a conceptual framework for understanding multiple proxy perspectives. Med Care. 2005;43:493-9.

20. le Coq EM, Boeke AJ, Bezemer PD, et al. Which source should we use to measure quality of life in children with asthma: the children themselves or their parents? Qual Life Res. 2000;9:625-36.

21. García-Altés A, Navas E, Soriano MJ. Evaluación económica de intervenciones de salud pública. Gac Sanit. 2011;25(Supl 1):25-31.

22. Burstrom K, Bartonek A, Brostrom EW, et al. EQ-5D-Y as a health-related quality of life measure in children and adolescents with functional disability in Sweden: testing feasibility and validity. Acta Paediatr. 2014;103:426-35.

23. Herdman M, Badia X, Berra S. El EuroQol-5D: una alternativa sencilla para la medición de la calidad de vida relacionada con la salud en atencion primaria. Aten Primaria. 2001;28:425-30.

24. Silverplats K, Lind B, Zoega B, et al. Health-related quality of life in patients with surgically treated lumbar disc herniation: 2- and 7-year follow-up of 117 patients. Acta Orthop. 2011;82:198-203.

25. Dolan $P$, Jones-Lee $M$. The time trade-off: a note on the effect of lifetime reallocation of consumption and discounting. J Health Econ. 1997;16:731-9.

26. Gusi N, Pérez-Sousa MA, Gozalo-Delgado M, et al. Validez y fiabilidad de la versión proxy del EQ-5D-Y en español. An Pediatr (Barc). 2014;81:212-9.

27. Cohen J. A power primer. Psychol Bull. 1992;112:155-9.

28. Tamim H, McCusker J, Dendukuri N. Proxy reporting of quality of life using the EQ-5D. Med Care. 2002;40:1186-95.

29. Badía X, Díaz-Prieto A, Rue M, et al. Measuring health and health state preferences among critically ill patients. Intens Care Med. 1996;22:1379-84.

30. Dinglas VD, Gifford JM, Husain N, et al. Quality of life before intensive care using EQ-5D: patient versus proxy responses. Crit Care Med. 2013;41:9-14.

31. Theunissen NC, Vogels TG, Koopman HM, et al. The proxy problem: child report versus parent report in health-related quality of life research. Qual Life Res. 1998;7:387-97. 